\title{
A model for explaining fusion suppression using classical trajectory method
}

\author{
C. K. Phookan ${ }^{1,2, a}$ and K. Kalita ${ }^{2, b}$ \\ ${ }^{1}$ Dept. of Physics, Haflong Govt. College, Haflong, India-788819 \\ ${ }^{2}$ Dept. of Physics, Gauhati University, Guwahati, India -781014
}

\begin{abstract}
We adopt a semi-classical approach for explanation of projectile breakup and above barrier fusion suppression for the reactions ${ }^{6} \mathrm{Li}+{ }^{152} \mathrm{Sm}$ and ${ }^{6} \mathrm{Li}+{ }^{144} \mathrm{Sm}$. The cut-off impact parameter for fusion is determined by employing quantum mechanical ideas. Within this cut-off impact parameter for fusion, the fraction of projectiles undergoing breakup is determined using the method of classical trajectory in two-dimensions. For obtaining the initial conditions of the equations of motion, a simplified model of the ${ }^{6} \mathrm{Li}$ nucleus has been proposed. We introduce a simple formula for explanation of fusion suppression. We find excellent agreement between the experimental and calculated fusion cross section. A slight modification of the above formula for fusion suppression is also proposed for a three-dimensional model.
\end{abstract}

\section{Introduction}

Reactions initiated by loosely bound projectiles like ${ }^{6} \mathrm{Li},{ }^{7} \mathrm{Li}$ and ${ }^{9} \mathrm{Be}$ have provided a new platform for the study of a rich repertoire of interesting phenomena [1,2]. These nuclei have unusually low separation energy among its constituents and therefore have a high breakup probability. The nucleus ${ }^{6} \mathrm{Li}$ breaks up into ${ }^{4} \mathrm{He}+{ }^{2} \mathrm{H}$ with separation energy, $S_{\alpha}=1.48 \mathrm{MeV}$. As a result these nuclei promptly break up during the fusion process of these projectiles with a heavy target nucleus. Here, at least four different types of events have been identified. When the whole of the projectile fuses with the target without breakup, then it is called direct complete fusion (DCF). After breakup, if both the fragments fuse with the target, then it is called sequential complete fusion (SCF). If one of the breakup fragments fuse with the target, then it is called incomplete fusion (ICF). If none of the breakup fragments fuse with the target, then it is called no-capture breakup (NCBU) [3]. Due to breakup, the experimental cross section falls below the theoretically expected value which is also called fusion suppression [2, 4, 5]. The calculation of breakup yields for reactions initiated by loosely bound projectiles has been done by CDCC as well as classical methods [6]. In the present work we shall use the classical trajectory method for an explanation of fusion suppression, and shall apply it to the reactions ${ }^{6} \mathrm{Li}+{ }^{152} \mathrm{Sm}$ and ${ }^{6} \mathrm{Li}+{ }^{144} \mathrm{Sm}$. Our method is based on Hagino's twodimensional classical trajectory method [7]. The essential idea is to determine the breakup fraction of the projectile as a function of the impact parameter. Then, by incorporating a quantum mechanical technique to find out the cut-o ff

a. e-mail: chinmoy.phookan@gmail.com

b. e-mail:ku_kalita@yahoo.com impact parameter for fusion [8], we propose a simple formula for the fusion suppression factor.

\section{Formalism}

We consider the projectile ( $\mathrm{Li}$ ) to be composed of a deuteron and an $\alpha$-particle. Hence, the Lagrangian for the system of projectile and target is,

$\mathcal{L}=\frac{1}{2} m_{1}\left(\dot{x}_{1}^{2}+\dot{y}_{1}^{2}\right)+\frac{1}{2} m_{2}\left(\dot{x}_{2}^{2}+\dot{y}_{2}^{2}\right)+\frac{1}{2} m_{3}\left(\dot{x}_{3}^{2}+\dot{y}_{3}^{2}\right)-V_{12}-V_{13}-V_{23}$

where, subscripts 1 and 2 denote the two projectile fragments, and subscript 3 denotes the target. $V_{i j}(i, j=1,2,3)$ denote the respective potential energies, which is composed of a Coulomb part and a nuclear part. The nuclear part is assumed to have a Woods-Saxon form. The twelve initial conditions needed for solving these equations are given below,

$$
\begin{array}{r}
x_{1}(0)=R-r_{1} \sin (\theta) \\
\dot{x}_{1}(0)=V+v_{1} \cos (\theta) \\
y_{1}(0)=b+r_{1} \cos (\theta) \\
\dot{y}_{1}(0)=-v_{1} \sin (\theta) \\
x_{2}(0)=R+r_{2} \sin (\theta) \\
\dot{x}_{2}(0)=V-v_{2} \cos (\theta) \\
y_{2}(0)=b-r_{2} \cos (\theta) \\
\dot{y}_{2}(0)=v_{2} \sin (\theta) \\
x_{3}(0)=0 \\
\dot{x}_{3}(0)=0 \\
y_{3}(0)=0 \\
\dot{y}_{3}(0)=0
\end{array}
$$


Here, $r_{1}, r_{2}$ and $v_{1}, v_{2}$ are respectively the distances and velocities of the projectile fragments (deuteron and $\alpha$ particle) with respect to their centre of mass (see Fig. 1). R is the initial distance of the centre of mass of the projectile from the target and $\mathrm{b}$ is the impact parameter. $\theta$ is the angle of orientation of the line joining the deuteron and the $\alpha$-particle with respect to the y-axis. Here, $\mathrm{V}=\sqrt{\left(2 E_{l a b}\right) / m}$ is the velocity of the projectile and is taken equal to the asymptotic velocity because for large $\mathrm{R}$ the Coulomb field is negligible. After repeated trial and error, we choose the

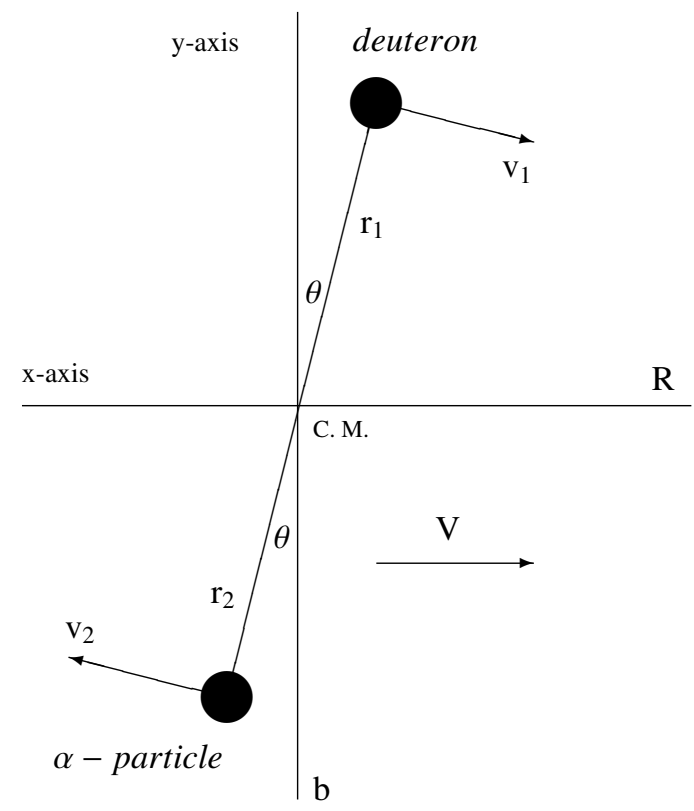

Figure 1. Model showing the cluster structure of ${ }^{6} \mathrm{Li}$. The centre of mass (C. M.) of the cluster is moving along the positive $\mathrm{x}$ axis with velocity $\mathrm{V}$. The $\mathrm{x}$ and $\mathrm{y}$ components of the distances of the C. M. from the target (not shown) are $\mathrm{R}$ and $\mathrm{b}$ (impact parameter), respectively.

values, $\mathrm{r}_{1}=2 \mathrm{r}_{2}=1.513 \mathrm{fm}, v_{1}=2 v_{2}=0.645 \times 10^{7} \mathrm{~m} . \mathrm{s}^{-1}$ because we could consistently obtain three types of trajectories and these are discussed in the next section. These values can be derived by considering a cluster model of ${ }^{6} \mathrm{Li}$. We consider the ${ }^{6} \mathrm{Li}$ nucleus to be composed of a deuteron and an alpha-particle, rotating in circular orbits about their centre of mass with a fixed distance of separation (see Fig. 1). We propose two postulates for the ${ }^{6} \mathrm{Li}$ cluster model : (a) The total energy is equal to the breakup threshold energy (binding energy) of the ${ }^{6} \mathrm{Li}$ nucleus, and (b) The total angular momentum of rotation of the deuteron and $\alpha$ particle about an axis through its centre of mass is equal to $\sqrt{\mathrm{I}(\mathrm{I}+1)} \hbar$, where I is the spin quantum number of the ${ }^{6} \mathrm{Li}$ nucleus. We would like to point out that in this semiclassical model of ${ }^{6} \mathrm{Li}$ the spin is manifested as an orbital motion, although predictions of the quantum mechanical model for the orbital angular momentum of $\alpha$-deuteron cluster for the ground state is $\mathrm{L}=0$ [9]. The details of the calculations are given in [3], where we obtain $2.27 \mathrm{fm}$ $\left(=r_{1}+r_{2}\right)$ as the distance between the deuteron and the $\alpha$-particle. Using the values of $\mathrm{r}_{1}, \mathrm{r}_{2}$ and $\mathrm{v}_{1}, \mathrm{v}_{2}$ as inputs, we obtain numerical solutions of the differential equations.
In order to find the cut-off impact parameter for fusion, we employ the method of one-dimensional quantum mechancial tunnelling over the fusion barrier. Assuming a sharp cut-off model for fusion, and using Hill and Wheeler's values of the transmission coefficients, we arrive at an expression for the fusion cross-section $\left(\sigma_{\text {fus }}\right)$ [8],

$$
\sigma_{f u s}=\frac{R_{b}^{2} \hbar \omega_{0}}{2 E_{c m}} \ln \left[\frac{1+e^{-y_{0}}}{1+e^{-z}}\right]
$$

where,

$$
z=y_{0}+\frac{\pi \hbar L_{c}\left(L_{c}+1\right)}{\omega_{0} R_{b}^{2} \mu}
$$

and,

$$
y_{0}=\frac{2 \pi\left(E_{0}-E_{c m}\right)}{\hbar \omega_{0}}
$$

Here, $\hbar \omega_{0}, \mathrm{E}_{0}$ and $\mathrm{R}_{\mathrm{b}}$ are the curvature, height and position of the Coulomb barrier, respectively. If these parameters are known, then Eq. (3) can be solved to find the cutoff angular momentum $\left(\mathrm{L}_{\mathrm{c}}\right)$ for every pair of $\sigma_{\text {fus }}$ and $\mathrm{E}_{\mathrm{cm}}$. Once $\mathrm{L}_{\mathrm{c}}$ is known, the cut-off impact parameter $\mathrm{b}_{\mathrm{c}}$ can be determined from the relation,

$$
b_{c}=\frac{L_{c} \hbar}{\sqrt{2 m E_{l a b}}}
$$

which is derived from the condition that the angular momentum of the projectile is $\mathrm{L}_{\mathrm{c}}$ times $\hbar$.

\section{Methodology for finding fusion suppression}

The methodology adopted by us is essentially using quantum mechanical laws for determining the cut-off impact parameter for fusion, and then using classical Newtonian laws for determining the relative number of projectile breakup. Classical laws tend to reproduce fairly accurate results only under certain conditions and they have their own limitations. One limitation is that the nature of the trajectory tend to change with the distance ( $\mathrm{x}$-component) of the projectile from the target. As such the distance $(\mathrm{R})$ is randomly varied between two limits in order to eliminate the effects of the dependence of the type of trajectory on R. We took the limits $350 \mathrm{fm}$ and $370 \mathrm{fm}$ as the Coulomb field is quite small compared to the total energy, and a difference of $20 \mathrm{fm}$ ensured that the projectile $\left({ }^{6} \mathrm{Li}\right)$ made a complete rotation as it moved through this distance. We keep $\theta$ constant at $0^{0}$ because it maximises the breakup fraction (defined below) compared to other angles. Changing $\mathrm{R}$ automatically changes the instantaneous $\theta$ at any position inside the two limits, and it would be a redundant exercise to change $\theta$ over all possible values. Some of the typical trajectories obtained from the numerical solutions are shown in Figs. 2, 3 and 4. Fig. 2 shows a scatteringlike event, Fig. 3 shows incomplete fusion in which the deuteron is captured by the target, whereas Fig. 4 shows no-capture breakup. Scattering-like events include complete fusion events (for events occuring inside the cutoff impact parameter) as well as actual scattering events (for events occuring outside the cut-off impact parameter) 


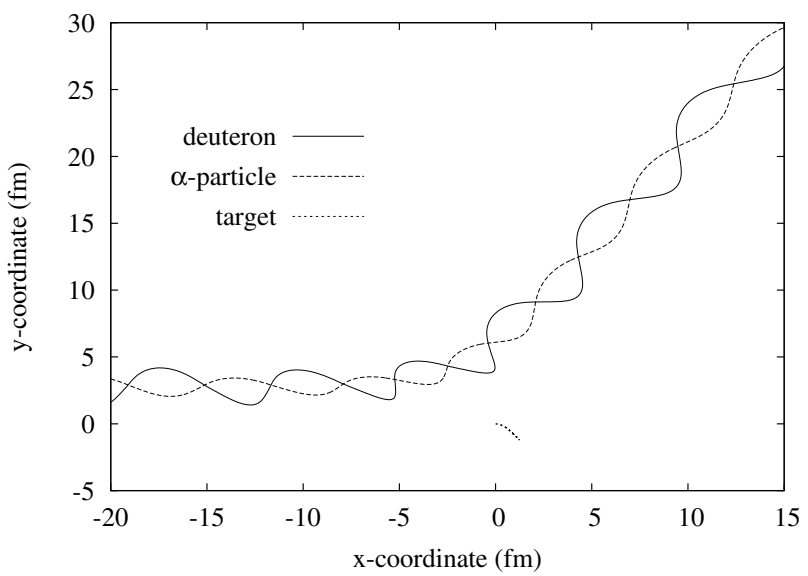

Figure 2. A scattering-like trajectory is shown which includes both scattering (for events above cut-off impact parameter) and complete fusion events (for events below cut-off imapct parameter).

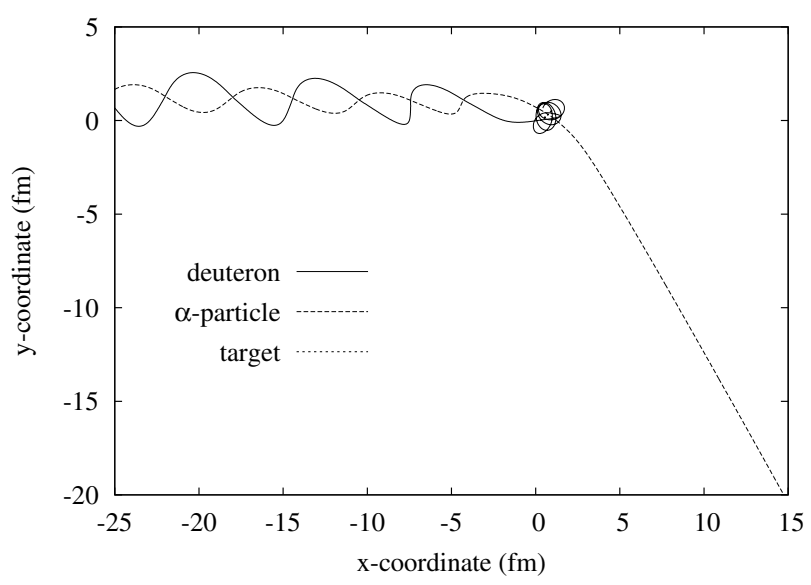

Figure 3. An incomplete fusion event is shown in which the deuteron is captured by the target.

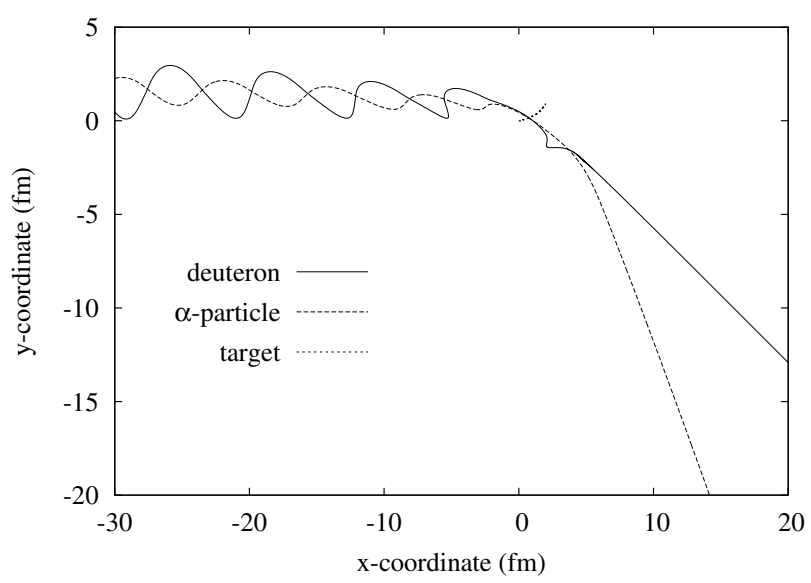

Figure 4. A no-capture breakup event is shown in which none of the projectile fragments are captured by the target.
[3]. And, complete fusion events include both direct complete fusion (DCF) and sequential complete fusion (SCF). We broadly classify the trajectories into two types, viz., breakup trajectory and no-breakup trajectory. The trajectories of Fig. $3 \& 4$ can be identified as a breakup trajectory, and the trajectory of Fig. 2 can be classified as a nobreakup trajectory. A breakup trajectory is identified from the condition that the distance of separation between the deuteron and the $\alpha$-particle is greater than $2.27 \mathrm{fm}$ after interaction with the target. The fraction of projectiles undergoing breakup will certainly depend upon the impact parameter. Hence, for a large number of trajectories we define the breakup fraction as,

$$
B_{i}=\frac{\text { number of trajectories that lead to breakup }}{\text { total number of trajectories }}
$$

where, subscript i denotes the impact parameter at which breakup fraction $\left(B_{i}\right)$ is evaluated. We define the fusion suppression factor as the average of the breakup fractions $\left(\mathrm{B}_{\mathrm{i}}\right)$ evaluated at different impact parameters with weightage given by the fusion probability $\left(\mathrm{P}_{\mathrm{i}}\right)$. The range of impact parameters starts from zero (i.e., a head-on collision) and is increased in steps of $0.2 \mathrm{fm}$ until the cut-off impact parameter $\left(b_{c}\right)$ is reached. Therefore,

$$
\text { Fusion Suppression }=\frac{\sum_{i} B_{i} P_{i}}{\sum_{i} P_{i}}
$$

For a sharp cut-off model,

$$
P_{i}=\left\{\begin{array}{lll}
1 & \text { if } & i \leq b_{c} \\
0 & \text { if } & i>b_{c}
\end{array}\right.
$$

In the above definition it is assumed that all breakup events leading to complete separation of the projectile are responsible for fusion suppression. Hence, events termed sequential complete fusion (SCF) which are more likely to occur inside the barrier are excluded. This is consistent with the empirical definition of fusion suppression,

$$
\mathrm{f}_{\text {exp }}=1-\frac{\sigma_{\text {exp }}}{\sigma_{\text {theo }}}
$$

The experimentally measured fusion cross section $\left(\sigma_{\exp }\right)$ is unable to make a distinction between direct complete fusion (DCF) and sequential complete fusion (SCF).

\section{Results and Discussion}

We shall be applying the above mentioned formalism to the reactions ${ }^{6} \mathrm{Li}+{ }^{152} \mathrm{Sm}$ and ${ }^{6} \mathrm{Li}+{ }^{144} \mathrm{Sm}$. Woods-Saxon parameters $V(v, r, a)$ of the nuclear potentials used are : $V_{d-\alpha}=(75.5 \mathrm{MeV}, 1.85 \mathrm{fm}, 0.71 \mathrm{fm}), V_{d-{ }^{152} \mathrm{Sm}}=(91.82$ $\mathrm{MeV}, 1.013 \mathrm{fm}, 0.938 \mathrm{fm}), V_{\alpha-{ }^{152} \mathrm{Sm}}=(60.5 \mathrm{MeV}, 1.107$ $\mathrm{fm}, 0.607 \mathrm{fm}), V_{d-{ }^{144} \mathrm{Sm}}=(99.72 \mathrm{MeV}, 1.15 \mathrm{fm}, 0.85 \mathrm{fm})$ and $V_{\alpha-{ }^{144} \mathrm{Sm}}=(185.0 \mathrm{MeV}, 1.40 \mathrm{fm}, 0.52 \mathrm{fm})$ [3]. For calculating the breakup fraction $\left(\mathrm{B}_{\mathrm{i}}\right)$, a sample of 50 trajectories are chosen for each impact parameter [3]. For determination of the empirical value of fusion suppression $\left(f_{\text {exp }}\right)$, we first determine the theoretical value of fusion 


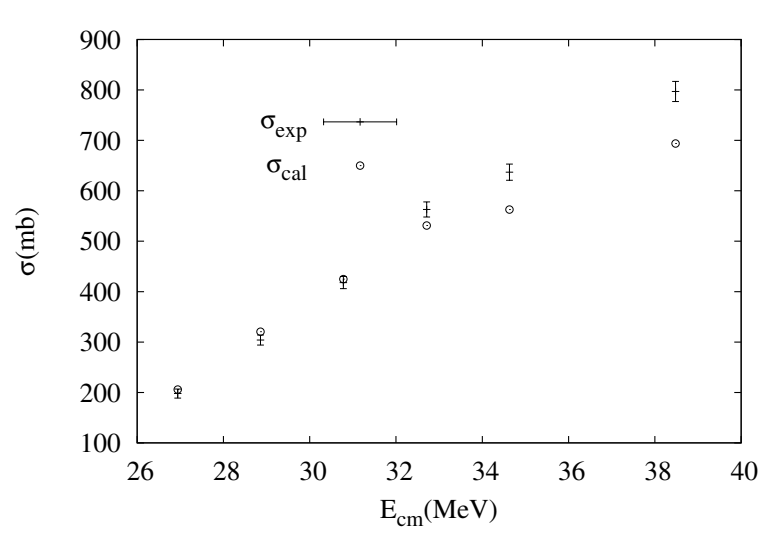

Figure 5. Calculated $\left(\sigma_{\text {cal }}\right)$ and experimental fusion cross section $\left(\sigma_{\text {exp }}\right)$ vs $\mathrm{E}_{\mathrm{cm}}(\mathrm{MeV})$ for the reaction ${ }^{6} \mathrm{Li}+{ }^{152} \mathrm{Sm}[4]$.

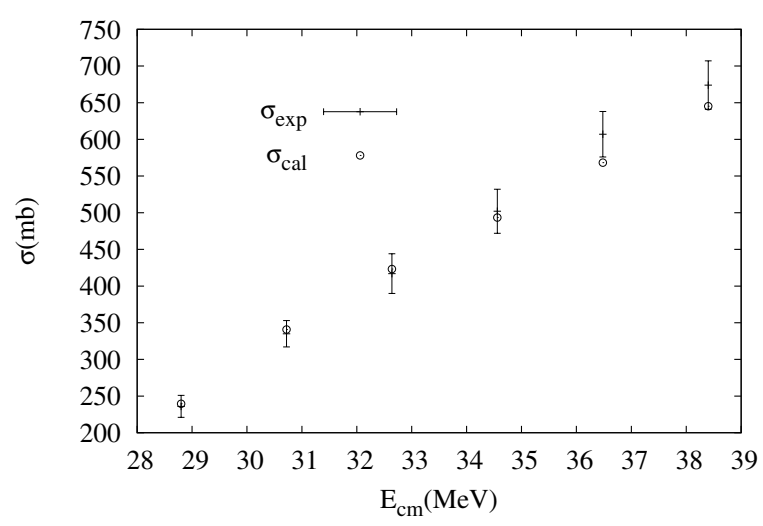

Figure 6. Calculated $\left(\sigma_{\text {cal }}\right)$ and experimental fusion cross section $\left(\sigma_{\text {exp }}\right)$ vs $\mathrm{E}_{\mathrm{cm}}(\mathrm{MeV})$ for the reaction ${ }^{6} \mathrm{Li}+{ }^{144} \mathrm{Sm}[5]$.

cross section ( $\left.\sigma_{\text {theo }}\right)$ which is expected in the absence of breakup. $\sigma_{\text {theo }}$ is determined by running CCFULL code $[3,10]$. Knowing the experimental value of fusion cross section $\left(\sigma_{\exp }\right)[4,5]$, the empirical fusion suppression factor is determined from Eq. (10) and the results are shown in tables 3 and 4 of Ref. [3]. Then, using Eq. (3), $\mathrm{L}_{c}$ is determined. Finally, from Eq. (6), the cut-off impact parameter $\left(b_{c}\right)$ is determined. Using the cut-off impact parameter $\left(b_{c}\right)$, the calculated value of fusion suppression $\left(f_{c a l}\right)$ is evaluated using Eq. (8), and the results are shown in tables 3 and 4 of Ref. [3]. From the $f_{\text {cal }}$ values, we determine the calculated fusion cross section $\left[\sigma_{\text {cal }}=\sigma_{\text {theo }}\left(1-\mathrm{f}_{\text {cal }}\right)\right]$. A comparison of $\sigma_{\exp }$ and $\sigma_{\text {cal }}$ is shown in Figs. 5 and 6 for the reactions ${ }^{6} \mathrm{Li}+{ }^{152} \mathrm{Sm}$ and ${ }^{6} \mathrm{Li}+{ }^{144} \mathrm{Sm}$, respectively. It can be concluded that there is very good agreement between the values of $\sigma_{\exp }$ and $\sigma_{\text {cal }}$. For ${ }^{6} \mathrm{Li}+{ }^{152} \mathrm{Sm}, \sigma_{\text {cal }}$ is slightly lower than $\sigma_{\text {exp }}$ for higher energies. This is because of the fact that the cut-off impact parameter $\left(b_{c}\right)$, as calculated from Eq. (6), is slightly lowered from the true value as SBPM values of $\sigma_{\text {fus }}$ are used in the calculations. In fact, for the system ${ }^{6} \mathrm{Li}+{ }^{144} \mathrm{Sm}, \sigma_{\text {cal }}$ values follow the exact trend of the $\sigma_{\text {exp }}$ (table 4 of Ref. [3]) values evaluated at different energies. The reason is that ${ }^{144} \mathrm{Sm}$ is least deformed, whereas ${ }^{152} \mathrm{Sm}$ is highly deformed $\left(\beta_{2}=0.26\right)$.

\section{Conclusion}

The agreement of our results with experimental data suggests that classical Newtonian laws are providing a fairly accurate picture for the breakup of ${ }^{6} \mathrm{Li}$ immediately above the barrier. Also, any dominant tranfer processes for the above reactions must be included under the breakup event described above. It would be interesting to see the validity of Eq. (8) for a three-dimensional model. In a three-dimensional model [6], the orientation of the projectile is not necessarily confined to a single plane which is the case for the two-dimensional model. In realistic three dimensions, the cross section would present an area (two-dimensions), but in two dimensions the cross-section would present a line (single dimension). Hence, in threedimensions we propose the following formula where a factor of $b_{i}$ (the impact parameter) is included in the numerator and denominator of Eq. (8). Hence,

$$
\text { Fusion Suppression }=\frac{\sum_{i}^{b_{c}} b_{i} B_{i} P_{i}}{\sum_{i}^{b_{c}} b_{i} P_{i}}
$$

where, the breakup fraction $\left(B_{i}\right)$ is evaluated at each impact parameter (i) and for all orientations of the projectile.

\section{References}

[1] C. K. Phookan and K. Kalita, Nucl. Phys. A, 899, 29 (2013).

[2] M. Dasgupta et al., Phys. Rev. C, 70, 024606 (2004).

[3] C. K. Phookan and K. Kalita, Journal of Physics G, 40, 125107 (2013).

[4] P. K. Rath et al., Nucl. Phys. A, 874, 14 (2012).

[5] P.K. Rath et al., Phys. Rev. C, 79, 051601 (2009).

[6] A. Diaz-Torres et al., Phys. Rev. Lett., 98, 152701 (2007).

[7] K. Hagino et al., Nucl. Phys. A, 738, 475 (2004).

[8] E. F. Aguilera and J. J. Kolata, Phys. Rev. C, 85, 014603 (2012).

[9] A. Diaz-Torres et al., Phys. Rev. C, 68, 044607 (2003).

[10] K. Hagino et al., Comp. Phys. Comm., 123, 143 (1999). 